\title{
Commentary
}

\section{COVID 19: a personal account of a physiotherapist}

\author{
Vandana Dua ${ }^{1 *}$, Puneet K. Dua ${ }^{2}$ \\ ${ }^{1}$ Department of Neurosciences, All India Institute of Medical Sciences, Ansari Nagar, New Delhi, India \\ ${ }^{2}$ Department of Physiotherapy, Indraprastha Apollo Hospital, Sarita Vihar, New Delhi, India
}

Received: 19 October 2020

Accepted: 08 January 2021

\author{
*Correspondence: \\ Dr. Vandana Dua, \\ E-mail: pt.vandana@gmail.com
}

Copyright: (C) the author(s), publisher and licensee Medip Academy. This is an open-access article distributed under the terms of the Creative Commons Attribution Non-Commercial License, which permits unrestricted non-commercial use, distribution, and reproduction in any medium, provided the original work is properly cited.

\begin{abstract}
Amidst the growing tentacles of coronavirus SARS2 in the densely populated Delhi (India), the healthcare workers and other essential service providers are working day and night to keep the city going. All are working with a fear, a fear of getting entrapped in the tentacles any moment. Here is a personal account of myself, a healthcare worker who got entrapped, thankfully not getting strangulated, having recovered early without getting trolled physically but certainly, psychologically, socially and emotionally. It is my first person account as being a patient myself, second person account as a caretaker of my family and a third person account as a therapist to my patients. Also, a note of physiotherapeutic management of COVID-19 is included.
\end{abstract}

Keywords: Chest physiotherapy, Coronavirus, First person account, Healthcare worker, Critical care

\section{INTRODUCTION}

I and my husband are physiotherapists, both are working in tertiary care setups serving COVID-19 patients. The tale begins on a Sunday noon, in the month of June 2020 when I was delivering an online lecture, the new normal of sharing expertise. I got a call from my workplace and I was being asked to isolate myself as I had fallen under a high risk of contracting COVID-19 after sharing my duty room with a colleague during my last duty who was found to be positive later that day. I immediately sent my kids to their uncle's place and locked myself in a room. My psychological stress began there itself; thoughts of getting sick, the guilt of unknowingly passing the infection to my loved ones, the fear of losing contact with them and the apprehension of being tabooed in the neighbourhood occupied me the whole night. I got up next morning with the news that my husband was feeling an unpleasant sensation in throat and vague body aches. We wondered if it was just psychosomatic or actually pathological. He developed low grade fever by evening. He was tested positive next morning and the first thing which came in our mind was the doubtful efficacy of eight week hydroxychloroquine (HCQ) prophylactic course we underwent being healthcare workers. Now our phone started ringing continuously with calls from our workplaces, community doctor, local police station, Arogya Setu, municipal corporation doctor, sub-district magistrate office etc. asking about his symptoms, likely source of infection, contact tracing, our decision of choosing home quarantine or hospitalization, online medical consultations, prescriptions and what not. With only mild symptoms of sore throat, dry cough, low grade fever and lethargy, the physical stress was bearable for him. By this time, I was busy sanitising the rest of house, taking care of his meals and other needs from outside the room with the constant fear of being under incubation period at the back of my mind. We were still trying to keep up a little humour alive in whatever little interactions we were having across the door until my mother was tested positive followed by me. Thankfully, all of us developed only mild symptoms and did not require hospitalization.

My husband suffered fever for 2 days, with sore throat that persisted for about a week. He felt weak for about 10 days and developed altered sensation of taste (hypogeusia) an smell (hyposmia) after a week of being positive, which gradually recovered over the next week. My symptom 
diary showed sore throat, low grade fever for 3 days, a weeklong myalgia, early fatigue and a delayed onset of anosmia and hypogeusia, which also normalised in a week span. The course for our medium-built 67 years old, hypothyroid, osteopenic, non-diabetic, and nonhypertensive mother was a little different. Though her fever subsided in 3-4 days, but myalgia continued for a long time. The most distressing issue was her depressed attitude with frequent episodes of weeping. We did temperature and oxygen saturation charting, gargles, took vitamin supplements and paracetamol SOS. We took steam inhalation for $10 \mathrm{~min}$ and did deep breathing exercises twice a day since the first day itself to maintain optimum ventilation. As soon as our fever subsided, we checked our functional capacity by six minutes' walk test. We tailored a 30 minute daily physical activity program including walking, stretching exercises and aerobics for all of us, to avoid any long standing physical issues. In addition, we incorporated various energy pacing strategies to our mother's routine to combat her body aches and low morale. The separation from our kids, social isolation, and managing household chores along with illness, were bringing negativity in our minds, while there was too much of positivity in our lab reports. While maintaining our physical activity routine, very soon we switched over to a cosy family time; watching web-series, video calling with our kids and having tea times together. My husband chose gardening as his pastime and I rediscovered my childhood hobby of painting. Our mother resolved to her religious routine, spending time listening to sermon. We waited eagerly for our quarantine period to get over.

The day finally arrived. We got our medical fitness and were liberated. We never knew the true sense of liberation until then. The walk in the lane around our house was never so satisfying. Welcoming the kids back home was no less than Diwali celebration for us.

Here onwards, I would take you to my journey as a therapist to COVID-19 patients. COVID positive patients in my hospital are primarily those who either have some or the other co-morbidity, and are hospitalised for their primary pathology or those who land up in emergency and found to be positive. For the ease of understanding, I would categorise the patients into mild, moderate and severe on the basis of severity of their respiratory involvement. The mild patients are the ones who are ambulatory, stable on their cardiopulmonary status and are maintaining oxygen saturation at room air. Moderate severity patients could be further subdivided into those who are maintaining their oxygen requirements either through low flow oxygen or the ones who require high flow oxygen/non-invasive ventilation. While the more severe ones in our set up include, the ones with one or more co-morbidity or may be immuno-compromised due to primary pathology and have increased viral load. Patients with cancer, long standing neurological or renal disorders and other bedridden patients form a major proportion among these. Most of them are mechanically ventilated, having developed acute respiratory distress syndrome
(ARDS) and shown rapid deterioration. Mild to moderate patients, here, are being taught to maintain optimum ventilation by 10 minutes of steam inhalation twice daily and 10-15 minutes of lung expansion sessions in form of deep diaphragmatic breathing, incentive spirometry is added after every 3 awake hours. They undergo a structured 15 min endurance activity twice daily and a progressive mobility program as per their performance on six minute walk test to maintain functioning and mobility. $\mathrm{L}$ test of functional mobility is being used for those who were more debilitated. They are monitored for their oxygen saturation, hemodynamics during the activity and are put on supplemental oxygen if required during/prior to the activity and the rest periods are given as per requirement. ${ }^{1}$ A routine of rotational positioning with emphasis to prone position is incorporated early in the disease course to prevent irreversible damage. The prone positioning leads to better lung recruitment, optimises ventilation-perfusion matching and hence has shown to improve oxygenation and prevent mortality in ARDS. ${ }^{2} \mathrm{~A}$ few patients, especially those with coexisting respiratory pathology, require assistance with airway clearance in form of forced expiratory techniques, positive expiratory pressure devices or if having altered sensorium, they require more passive techniques in form of percussions, vibrations or suctioning. 12-14 hours of prone positioning, maintaining bronchial hygiene, preventing complications like pressure sores, muscle contractures, and deep vein thrombosis remain the mainstay of physiotherapy in most severe ones. ${ }^{3}$

The patients in the recovering phase face long standing low grade fever; sleep deprivation, cardiac issues including arrhythmias, myocarditis, myocardial infarction, postural hypotension; renal dysfunction; gastrointestinal upsets in form of diarrhoea and vomiting; neurological issues like mental confusion, Gullian Barre syndrome, stroke; cognitive and psychological issues; respiratory issues ranging from frequent coughs, breathlessness to progressive lung fibrosis. They also face long standing fatigue, myalgia, low energy levels, low self-esteem, balance issues, poor cardiopulmonary endurance, post critical-care weakness which is effectively managed by physiotherapists. The vital role of inspiratory muscle training, lung expansion techniques, airway clearance strategies, individualised strength and endurance training, pacing strategies, postural correction, balance and gait training cannot be overemphasized.

All the hospitalised COVID-19 patients, without exception, face anxiety, fear of progression of disease, pain of separation from their family and uneasiness of being in an unknown environment with everyone donned in this awkwardly looking personal protective equipment (PPE). I realised that even a 2 minute counselling or simply listening to them does wonders in their psychological and emotional status, which undoubtedly transforms into better compliance to therapy. I am thankful to almighty that I have now become more sensitive to them. My work starts with a prayer to strengthen me so as to enable me to help 
my patients. But working with PPEs has its own limitations. The uncomfortable attire, heavy face shield, constant pressure on back of ears and bridge of nose, the drooling sweat all over the body, dehydration, suffocation and headache due to breathing in the masks for long hours, the blurred vision due to fogged eye gears, and controlling the nature's calls are just a few to mention. Recently, 'the anti-fog restorer' often used by swimmers came to our rescue against fogging.

In addition to this physical stress, we healthcare workers also have our own share of psychological stress. We face the fear to contract the infection to an extent that being finicky about washing and sanitising hands and equipments, looking every colleague around with suspicion of being infected and relating every ache or symptom of own or others around to be a symptom of coronavirus infestation. The pain of losing colleagues and patients are constantly bogging us down. The apprehension of carrying the infection to our homes is quite natural. The regular household chores including cooking for family, nursing the elderly at home or nurturing our little ones is now not free of the thought that we might be silent carriers of infection. The office conversations are full of COVID-19 news, predictions, news of heightened risks in healthcare professionals and even rumours, all playing with our psychology. Many of us have also faced social ostracism, so much so that our families faced social boycott and our kids couldn't play with their peers around. I wonder at times, how this era has pushed a social animal like man into solitude where each one is thinking of one's own safety and well-being.

A positive mental attitude, taking frequent break offs, maintaining a daily routine of physical exercise, meditation, deep breathing exercises, healthy diet, sound sleep schedule, talking to peers, working in partnerships, verbalising ones fears, self-monitoring, regular health check -ups are a few strategies which are effectively enabling us to move on in this hardship. This COVID era has taught me to filter the plethora of news and focus only on positive news. Fear of 'fear' is more fearful than fear itself. This proverb stands true in this time too. Pessimistic attitude, uncertainty about future and doubting selfcapabilities initiate a cascade of negativity, inability to take decisions and depressive attitude affecting the coping mechanism. Thanks to my colleagues and friends who always motivated me to maintain optimism, focusing on inner strength to cope up.
At the same time, like any other thing around the globe, these times also have a positive aspect to it. It has undoubtedly slowed the fast paced lives, brought the families together, and made us realise the difference between luxury and necessity in almost every domain of our lives. We have resolved to a healthier lifestyle with better hygiene practices. Our society has witnessed exemplary anecdotes of a lot of volunteers working selflessly day and night for the needy. The rapid technological advancement around, has further strengthened the notion that 'need is the mother of any invention'. Still, I wish and pray that if someone could uninstall '2020' or get an antivirus to debug our system from this virus and bring back our dynamic social lives full of fun and frolic.

\section{ACKNOWLEDGEMENTS}

Authors would like to thank Dr. Hemanshu Prabhakar, Professor, Department of Neuro-Anaesthesia, A.I.I.M.S., New Delhi for the critical revision of the manuscript.

Funding: No funding sources

Conflict of interest: None declared

Ethical approval: Not required

\section{REFERENCES}

1. Garvey C, Bayles MP, Hamm LF, Hill K, Holland A, Limberg TM, Spruit MA. Pulmonary Rehabilitation Exercise Prescription in Chronic Obstructive Pulmonary Disease: Review of Selected Guidelines: An Official Statement From The American Association Of Cardiovascular And Pulmonary Rehabilitation. J Cardiopulm Rehabil Prev. 2016;36(2):75-83.

2. Munshi L, Fralick M, Fan E. Prone positioning in non-intubated patients with COVID-19: raising the bar. Lancet Respir Med. 2020;8(8):744-5.

3. "Guidelines for Physiotherapy Practice in COVID crisis". Maharashtra State council for occupational therapy and physiotherapy, Mumbai. 2020. Available at: www.msotptcoucnil.com. Accessed on: 09 June 2020 .

Cite this article as: Dua V, Dua PK. COVID 19: a personal account of a physiotherapist. Int J Sci Rep 2021;7(2):140-2. 